\title{
Merger of Compact Binaries in the Context of Gravitational Waves and Short-Lived Gamma-Ray Bursts
}

\author{
Shawqi Al Dallal1, Walid J. Azzam² \\ ${ }^{1}$ College of Graduate Studies and Research, Ahlia University, Manama, Bahrain \\ ${ }^{2}$ Department of Physics, College of Science, University of Bahrain, Zallaq, Bahrain \\ Email: shaldallal@gmail.com
}

How to cite this paper: Al Dallal, S. and Azzam, W.J. (2018) Merger of Compact Binaries in the Context of Gravitational Waves and Short-Lived Gamma-Ray Bursts. Journal of Modern Physics, 9, 2233-2256.

https://doi.org/10.4236/jmp.2018.912141

Received: September 20, 2018

Accepted: October 19, 2018

Published: October 22, 2018

Copyright $\odot 2018$ by authors and Scientific Research Publishing Inc. This work is licensed under the Creative Commons Attribution International License (CC BY 4.0).

http://creativecommons.org/licenses/by/4.0/

\begin{abstract}
The discovery of gravitational waves resulting from the merger of two massive black holes (GW150914) has revolutionized our view of merging compact binaries. Recently, the Swope Supernova Survey of the optical counterpart of a gravitational wave event in the NGC 4993 galaxy, GW170817, emanating from the merger of two neutron stars, has triggered a lot of research work. Emphasis has been on comparing the existing theoretical models with the observational data, allowing for the prospect of an even more stringent test of general relativity. The afterglow of this event was observed in a wide range of wavelengths extending from radio waves to gamma rays. In this work, we first explore the evolutionary pathways of compact binary systems following the in-spiral, merger, and ring down sequence. We then proceed to discuss the processes leading to the production of gravitational waves and electromagnetic emission resulting from the merger of compact objects, particularly neutron star binaries and neutron star-black hole systems. We construct a basic inventory of the energy released during the merger of compact binaries in all bands of the electromagnetic spectrum with emphasis on gamma-ray burst emission. The constraints on certain wavelength emissions, such as gamma-ray bursts, are discussed in terms of orbital dynamical instabilities, energy transfer processes, and possible jet orientations with respect to the observer. Finally, we explore the futuristic perspective of the impact of gravitational waves detection on our understanding of the working of the universe.
\end{abstract}

\section{Keywords}

Merging Binaries, Compact Objects, Gravitational Waves, Gamma-Ray Bursts 


\section{Introduction}

Gravitational waves are ripples in the fabric of spacetime generated by accelerating masses. They are generated also by a change in the quadruple moment, which is produced only when there is an asymmetrical movement of mass. They propagate outward as a wave from their source with the speed of light, and transport energy as gravitational radiation.

The possibility of the existence of gravitational waves (GW) was discussed in 1893 by Oliver Heaviside evoking the analogy between the inverse square law in electromagnetism and gravitation [1]. In 1905, Henri Poincaré proposed their existence as being required by the Lorentz transformation, and suggested that, in analogy to accelerating electrical charge emitting electromagnetic waves, an accelerating mass should generate GWs [2]. Subsequently, gravitational waves were predicted in 1916 by the general theory of relativity [3] [4].

Gravitational waves can provide deeper insight into important natural phenomena in the universe. They can penetrate regions of space prohibited to electromagnetic radiation. Thus, they allow, for example, the observation of the merger of compact objects in remote galaxies billions of light years away. Gravitational waves can also be used to probe the very early universe before the era of recombination when the universe was opaque to electromagnetic radiation. Primordial gravitational waves were predicted by the theory of inflation during the very early universe when spacetime experienced a short period faster than light expansion. However, this background signal is very weak to be observed by current instruments.

An observer of a GW will discover that spacetime is distorted by the effect of strain. This effect will produce a rhythmic increase and decrease of distances between objects at the frequency of the wave, and its magnitude decreases in proportion to the inverse square law from the source. However, this effect when measured on Earth after propagating through astronomical distances is extremely small, having strains of the order $10^{-21}$. This strain is within the accuracy limit of the LIGO and Virgo instruments [5].

The first indirect evidence of the existence of GWs was discussed by Russell Alan Hulse and Joseph Hooton Taylor. They discovered the first binary pulsar in 1974 [6]. The characteristics of the orbit can be inferred from the Doppler shifting of radio signals generated by the pulsar. Accumulated data in 1979 showed a gradual decay of the orbital period fitting precisely the expected loss of energy and angular momentum as predicted by the general theory of relativity.

In the first part of this work, we introduce the theoretical framework characterizing gravitational waves produced by the merger of compact binaries. In the second part, we proceed to discuss evolutionary pathways leading to the formation of compact binaries. In the third part, the processes and constrains involved in the merger of compact binaries are discussed. In the fourth part, the methods of detection of gravitational waves are introduced. This is followed by describing real merger events of black hole binaries and neutron star binary systems. In the 
last part of this paper we explore the futuristic perspective of gravitational waves and their impact on new horizons in astrophysics.

\section{Parameters Characterizing Gravitational Waves}

In this section we introduce some parameters describing the behavior of gravitational waves generated by the merger of compact binaries. These parameters will be helpful in shedding light on the nature of the processes leading to the merger of compact binaries. Let us first consider the merger of NS-NS binaries at a separation of " $a$ ". External perturbation may cause the decay of their orbits, until they merge, at a rate of $\mathrm{d} a / \mathrm{d} t=-a / \tau_{G W}$, where $\tau_{G W}$ is the gravitational merger timescale. For circular binary orbit decay, and assuming both NS can be approximated as point masses, the gravitational merger timescale is given by [7]:

$$
\tau_{G W}=\frac{5}{64} \frac{c^{5}}{G^{3}} \frac{a^{4}}{\mu M^{2}}=\frac{5}{64} \frac{c^{5}}{G^{3}} \frac{a^{4}}{q(1+q) M_{1}^{3}}
$$

Employing geometrized units ( $G=c=1$ ), the above equation can be written as:

$$
\tau_{G W}=2.2 \times 10^{8} q^{-1}(1+q)^{-1}\left(\frac{a}{R_{\odot}}\right)^{4}\left(\frac{1.4 M_{\odot}}{M_{1}}\right)^{3}
$$

In the above equations $M_{1}$ and $M_{2}$ are the individual NS masses, $M$ is the combined total mass $\left(M=M_{1}+M_{2}\right), \mu=M_{1} M_{2} / M$ is the reduced mass, and $q=M_{2} / M_{1}$ is the binary mass ratio, $c$ is the velocity of light, $G$ is the gravitational constant, and $M_{\odot}$ and $R_{\odot}$ are the solar mass and the solar radius, respectively. For an elliptical orbit, $\tau_{G W}$ is shorter, and the eccentricity is gradually reduced by the emission of gravitational waves, until circularization is achieved as they decay. The merger lifetime can be obtained by integration as $\tau_{\text {merger }}=\tau_{G W} / 4$. The luminosity of the emitted gravitational waves by NS binary systems is given by [7]:

$$
\begin{aligned}
L_{G W} & =\frac{32}{5} \frac{\mu^{2} M^{3}}{a^{5}}=\frac{32}{5} \frac{M_{1}^{2} M_{2}^{2}\left(M_{1}+M_{2}\right)}{a^{5}} \\
& =5.34 \times 10^{32} q^{2}(1+q)\left(\frac{M_{1} R_{\odot}}{1.4 M_{\odot}}\right)^{5} \frac{1}{a^{5}} \mathrm{erg} / \mathrm{s}
\end{aligned}
$$

At the end of the binary lifetime, the distance between its components shrinks to within a few NS radii, and the luminosity increases enormously, as predicted by the above equation, approaching the luminosity of all visible matter in the universe $\left(\approx 10^{53} \mathrm{erg} / \mathrm{s}\right)$.

The strain amplitude $h$ observed face-on at a distance $D$ from the source can be approximated by [7]:

$$
h=\frac{4 M_{1} M_{2}}{a D}=5.53 \times 10^{-3} q\left(\frac{M_{1}}{1.4 M_{\odot}}\right)^{2}\left(\frac{a}{100 \mathrm{~km}}\right)^{-1}\left(\frac{D}{100 M_{p c}}\right)^{-1}
$$

LIGO and Virgo instruments are sensitive enough to observe strain ampli- 
tudes of the order $10^{-21}$. The corresponding gravitational wave characteristic frequency is given by:

$$
f_{G W}=2 f_{\text {orb }}=\frac{1}{\pi}\left(M / a^{3}\right)^{1 / 2}=194\left(\frac{M}{2.8 M_{\odot}}\right)^{1 / 2}\left(\frac{a}{100 \mathrm{~km}}\right)^{-3 / 2} \mathrm{~Hz}
$$

Again, the frequency increases appreciably as the distance between the NS binary shrinks to a few NS radii. Gravitational waves are expected to have a wide range of frequencies: $10^{-16} \mathrm{~Hz}<f<10^{4} \mathrm{~Hz}$ [8].

An interesting measurement that can be achieved with direct GW observations, is the orbital decay rate, with the period evolving according to the relation:

$$
\frac{\mathrm{d} T}{\mathrm{~d} t}=-\frac{192 \pi}{5}\left(M_{C} \omega\right)^{5 / 3}
$$

where $\omega$ is the angular frequency, and $M_{C}$ is the chirp mass given by [7] [9]:

$$
M_{C}=\mu^{3 / 5} M^{2 / 5}=\frac{\left(M_{1} M_{2}\right)^{3 / 5}}{\left(M_{1}+M_{2}\right)^{1 / 5}}=\frac{c^{3}}{G}\left[\frac{5}{96} \pi^{-8 / 3} f^{-11 / 3} \dot{f}\right]^{3 / 5}
$$

This is a parameter that can be determined readily from GW observations.

\section{Evolutionary Pathways for Compact Binary Formation}

Merger of neutron stars or neutron star-black whole (BH) systems, with merger timescales smaller than the Hubble time, are typically formed via similar evolutionary pathways in stellar-field galaxy populations [10]. Several scenarios were proposed for the formation of compact binaries. In one scenario, the standard channel for NS-NS or BH-NS merging binaries is that the first-born compact object passes through a common envelope (CE) phase. Other models have also been proposed. One of these models assumes that the progenitor binary stars have nearly equal mass, and that they evolve off the main sequence and enter a CE phase prior to either undergoing a supernova [11] [12]. Simulation of this latter process shows that NS-NS systems could be formed by twin giant stars with core masses $\geq 0.15 M_{\odot}$. The standard pathway assumes a high-mass progenitor binary with both stars having a mass in the range $M \geq 8-10 M_{\odot}$ to ensure the burst of a pair of supernovae [11] [12]. In this system, the most massive star evolves over just a few million years before leaving the main sequence. It then passes through a giant phase and undergoes a Type Ib, Ic, or II supernova producing what will become the heavier compact object: a BH in BH-NS systems, or the most massive NS in NS-NS systems. Subsequently, the secondary star leaves the main sequence and enters a CE phase as it evolves to the giant phase, overflowing its Roche lobe [7]. The binary separation then shrinks dramatically as a result of dynamical friction until sufficient energy is produced to expel the envelope. This is an important step to keep the binary compact objects close enough through the emission of the gravitational waves within the Hubble time. In this scenario, the exposed helium-rich core of the secondary star undergoes a supernova with the consequence of either unbinding the system or producing a tight binary. The outcome of this process depends on the magnitude and orien- 
tation of the supernova kick.

The implications of the above scenarios:

- Accretion of matter by a NS has a limit beyond which accretion-induced collapse may take place [11]. In fact, the CE efficiency as determined by the range of binary separation and the mass of the primary compact object following the accretion phase, is very poorly constrained [9] [13] [14] [15].

- The relation between the initial star mass and the final compact object mass is fairly understood. However, uncertainties may arise from poor knowledge of the metallicity, which is a crucial factor when considering the effect of mass loss in stellar winds [16] [17].

- The fate of the system after the merger is determined by the maximum mass of the newly formed NS. It either undergoes accretion-induced collapse to a $\mathrm{BH}$ or survives as a NS. At present, a maximum mass of $1.97 \pm 0.04 M_{\odot}$ was obtained for a NS by the Shapiro time delay measurements [18]. GW observations may provide further constrains [19] [20] [21]. Supernova remnants may indicate whether the NS has a classic hadronic composition or instead consists of some form of strange quark matter or other particle condensates [22] [23] [24] [25]. On the other hand, the supernova kick velocity distribution is not fully understood. This is an important issue that determines whether the system becomes unbound or remains bound after the second explosion [23] [24] [25] [26]. Figure 1 summarizes the various scenarios of the evolutionary pathways for binary compact object formation.

Many population synthesis models have been proposed to understand binary system evolution in our Milky Way Galaxy. Assumptions about the CE evolution, the supernova kick distribution, and other parameters have been advanced. In certain models, population synthesis is normalized by estimating the star formation history in the Milky Way Galaxy [27] [28]. Other works involve the choice of the best judged parameters capable of reproducing the observed Galactic binary pulsar sample [29] [30].

A quantity of interest is the number of BH-NS and NS-NS mergers. Interferometric detection has a high sensitivity to the chirp mass $M_{C}$ (see Equation (7)), and to the binary mass ratio $q$ [31] [32] [33] [34]. If the signal to noise ratio is sufficiently high, it is possible to determine whether the primary's mass exceeds the maximum mass of a NS, even for frequencies outside the range of the LIGO band [7].

\section{Merger of Compact Stars Binaries}

The merger of compact binaries follows roughly three phases: in-spiral, merger, and ring down. Each of these phases constitutes a set of challenges for numerical modeling and detection.

The quazi-equilibrium (QE) formalism describes accurately the binary in-spiral phase, up until the point where the gravitational radiation timescale becomes comparable to the dynamical timescale. When the binary separation 


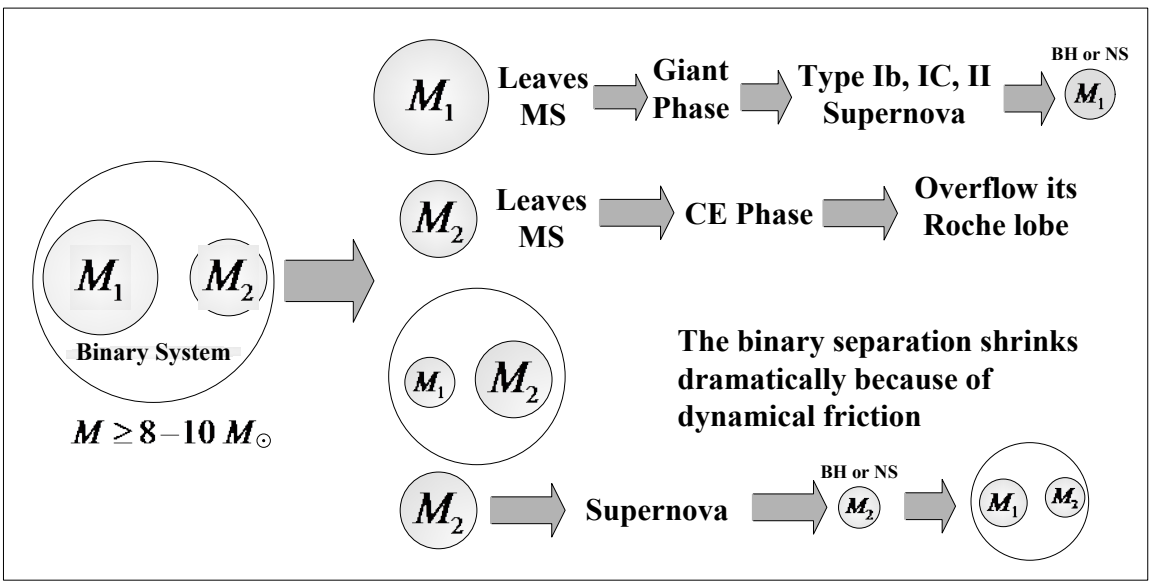

Figure 1. Evolutionary pathways of compact binaries formation.

shrinks to a few radii of the two NSs, the binary system becomes rapidly unstable. Dynamical instability causes the neutron stars to plunge and enter the merger phase. This is a complicated hydrodynamic phase that requires full GR simulations to be fully understood. Simulations show that if the NSs are of equal mass, the merger resembles a slow collision. On the other hand, if the primary is substantially more massive, then the secondary will be tidally disrupted during the plunge phase and will accrete onto the primary. Prior to merging, the NSs are most likely irrotational, leading to a substantial velocity discontinuity at the surface of contact and giving rise to a rapid production of vortices [7]. Meanwhile, a disk will form around the central remnant from the material that escapes from the outer Lagrange points of the system. Numerical simulation predicts that this phase produces the maximum GW amplitude. Gravitational waves during merger provide important information about the NS equation of state (EOS); in particular, it provides information about the gravitational wave frequency $f_{\text {orb }}$ at which the binary orbit becomes unstable (see Equation (5)). The merger may generate the thermal energy necessary to power the short-lived gamma-ray bursts (sGRB). This occurs when neutrinos and antineutrinos produced by the shock-heated material enter an annihilation phase around the remnant, producing high-energy gamma-ray photons.

In the ring-down phase, the system is finally settled in a dynamically stable configuration. The GW signal depends in this case on the remnant's mass and rotational profile. If the remnant is massive enough, it becomes gravitationally unstable, and it collapses to form a spinning BH. Alternatively, three possible scenarios are expected, depending on the total mass of the remnant [7]:

- If the remnant mass is less than the maximum mass of an isolated $\left(M_{i s o}\right)$ nonrotating NS, then it will survive and become stable forever.

- A supermassive remnant with a mass exceeding the isolated stationary mass limit, but below that allowed for a uniformly rotating NS (typically $\leq 1.2 M_{\text {iso }}$, and assuming weak dependence on the ESO), may become unstable [35] [36] [37]. Supermassive remnants are usually stable against gravita- 
tional collapse, unless angular momentum losses drive the angular velocity below the allowed value for stability. Angular momentum losses may originate from pulsar-type emission or magnetic coupling to the outer disk.

- A remnant entering the hypermassive regime, may be supported by a rapid differential rotation. Hypermassive NS remnants (HMNR) may have significantly larger masses, depending on the EOS, and will survive for a timescale much larger than the dynamical time, exhibiting a wide range of oscillation modes [38] [39] [40] [41] [42]. Eventually, differential rotation decreases gradually due to some combination of radiation reaction and magnetic viscous dissipation resulting in a collapse of the HMNS to a spinning black hole. The energy released from the collapse to HMNS may cause a delay in the sGRB, and the peak of the GW emission, powered by the collapse of the HMNS into a $\mathrm{BH}$, occurs only at a later time. The discrepancy in the time of arrival between the GW and the sGRB was observed for the first NS-NS merger detected by LIGO (see sec. 7.2.3).

Calculations have shown the possible formation of gravitationally-bound thick disks of material around the remnants. These types of disks are expected to heat-up appreciably, possibly yielding electromagnetic emission. However, these disks are not GW emitters, because of their relatively axisymmetric configuration and their inherently low densities. Under certain circumstances, a gravitational unbound outflow from mergers may occur. This outflow may be the site of exotic nuclear reactions produced by the $r$-process and leading to the formation of heavy elements.

\section{Merger of Black Hole-Neutron Star Binaries}

In-spiral of BH-NS system can also be described by post-Newtonian (PN) expansion, up until shortly before merger [7]. However, the parameter space is profoundly different from the NS-NS case. In the first place, a BH is heavier than a NS, and consequently the dynamics can follow a different pathway. Furthermore, BHs may be spinning rapidly, and the spin-orbit coupling can play a decisive role in the orbital dynamics of the binary giving rise to a larger number of oscillation modes in the GW signal [43] [44]. The larger mass of BH-NS binaries implies that instability occurs at a lower GW frequency (see Equation (5)). The instability of BH-NS binary systems is determined primarily by the binary mass ratio, NS compactness, and to a lesser extent by the $\mathrm{BH}$ spin [45]. Generally, the binding energy assumes its lowest value as the radius increases. For systems with high $\mathrm{BH}$ masses and/or more compact NS, the system is driven to dynamical orbital instability that occurs near the classical innermost stable circular orbit. In this case, the NS plunges toward the BH before the onset of tidal disruption, and the NS is typically swallowed as one piece by the $\mathrm{BH}$ with no material left behind to form a disk [7]. The GW emission from such systems is severely reduced after the merger event, leaving only a low-amplitude, rapidly decaying ringdown signal [46]. 
An interesting case to consider occurs when the NS and the $\mathrm{BH}$ have a mass ratio close to unity, the NS is less compact, and the $\mathrm{BH}$ has a prograde spin direction. In this case, the NS will reach the mass-shedding limit prior to dynamical instability, leading to its tidal disruption. The in-spiral is immune to angular momentum transfer via mass-shedding, and has never been seen in full or even approximate GR calculations [47]. However, unstable mass transfer may lead to the formation of a substantial disk around the $\mathrm{BH}$. In this process, the NS matter is accreted promptly by the $\mathrm{BH}[45]$.

\section{Detection of Gravitational Waves}

In the late 1950s, the scientific community was focused on whether GWs could transmit energy. Richard Feynman settled this question by a thought experiment known as the "sticky bead argument", presented during the First Gravitational Relativity Conference at Chapel Hill in 1957. In this argument, he noted that the effect of passing a GW on a rod with beads would be to move the beads along the rod, and consequently heat is generated, implying that GWs produce work. The Chapel Hill Conference inspired Joseph Weber to start designing and building a GW detector, known as the Weber bar, which is a large solid bar isolated from environmental vibrations. During the period 1969-1970, he claimed detecting gravitational waves emanating from the Galactic Center. However, the rate of this detection raised doubts on the validity of his observations, as this would drain our galaxy of energy at a time scale shorter than its inferred age. These doubts were further confirmed by other research groups. By the late 1970s, there was a general consensus that Weber's observations were spurious.

Modern GW detectors employ interferometry to achieve the required detection sensitivity. The most sensitive detectors using this scheme are the LIGO instruments at Hanford and Livingstone, and the Virgo instrument in Italy. These instruments are sensitive to strains of the order $h=10^{-21}$. One of the most promising future GW detection instruments is under development at Chongqing University. It is planned to detect high frequency gravitational waves with typical parameters: $f=100 \mathrm{GHz}$ and $h=10^{-31}$ to $10^{-32}$ [48].

The LIGO and Virgo detectors are modified Michelson interferometers. GW strain is measured as the difference in length between its orthogonal arms. Each arm has two mirrors at its ends and is $4 \mathrm{~km}$ in length. A passing $\mathrm{GW}$ alters the arm length $(\Delta L)$ in the $x$ and $y$ directions, such that, $\Delta L=h L$, where $h$ is the GW strain amplitude. This change in length produces a phase difference between the two laser beams passing through the beam splitter, and thus transmits an optical signal proportional to the GW strain to the output detector. To achieve the required sensitivity, each arm contains a resonant optical cavity formed by two test mass mirrors, which multiplies the effect of the GW on the light phase by a factor of 300 [49]. Additional resonant buildup of the laser light in the interferometer is achieved by placing a partially transmitting power-recycling mirror at the input [50]. Thus, the $20 \mathrm{~W}$ of laser input is increased 
to $700 \mathrm{~W}$ incident on the laser beam splitter, which is further increased to 100 $\mathrm{kW}$ that circulates in each arm cavity. Furthermore, the GW signal is optimized by placing a partially transmitting signal-recycling mirror at the output. This allows the extraction of the signal by broadening the bandwidth of the arm cavities [51] [52]. Figure 2 illustrates the modified Michelson interferometer used for GW detection.

\section{Observational Evidence of Gravitational Waves Emanating from the Merger of Compact Binaries}

The completion of the LIGO and Virgo detectors has enabled the scientific community, for the first time, to detect gravitational waves. In the following sub-sections, the characteristics of the detected gravitational waves and their progenitors are discussed.

\subsection{Gravitational Waves from Binary Black Hole Merger}

On September 14, 2015, the LIGO Hanford, WA, and Livingstone, LA, Observatories detected the first signal carrying the signature of a black hole binary merger. A schematic diagram of the gravitational wave signal of this event is shown in Figure 3. In this figure, the strain $h\left(10^{-21}\right)$ is shown as a function of time (s). Over $0.25 \mathrm{~s}$ the frequency increases from $35 \mathrm{~Hz}$ to $150 \mathrm{~Hz}$, where the amplitude of the signal assumes its maximum value. This type of signal is typical of gravitational waves emanating from the merger of compact binary stars. For lower frequencies, the event is characterized by a chirp mass given by Equation (7). Figure 3 provides an estimation of $f$ and $\dot{f}$, leading to a chirp mass of $M_{C} \approx 30 M_{\odot}$, and implying a total mass of the binary compact stars $M=M_{1}+M_{2} \geq 70 M_{\odot}$ [53]. The corresponding sum of the Schwarzschild radii of the binary components is $2 G M / c^{2} \geq 210 \mathrm{~km}$. At a frequency of $75 \mathrm{~Hz}$, which is half the maximum gravitational wave frequency, the binary objects must be very close and very compact [53]. A distance of $350 \mathrm{~km}$ is typical for equal Newtonian point masses orbiting at this frequency.

A chirp mass of $30 M_{\odot}$ cannot be obtained for a pair of two neutron stars. Moreover, a BH-NS binary having the deduced chirp mass would merge at a much lower frequency. Therefore, the merger of a BH binary is the only known system that can reach an orbital frequency of $75 \mathrm{~Hz}$ [53]. The damping oscillation of the signal (see Figure 3), after reaching its maximum value, is consistent with a black hole relaxing to a final stationary Kerr configuration [53].

Using general relativity-based models [54] [55] [56] [57], and performing a coherent Bayesian analysis to derive the posterior distribution of the source parameter [58], the initial and final masses, final spin, distance, and redshift of the source are obtained [53].

Fitting numerical simulations of binary black hole mergers [59] [60], allows the estimation of the mass and spin of the final black hole, the total energy radiated in gravitational waves, as well as the peak gravitational wave luminosity 


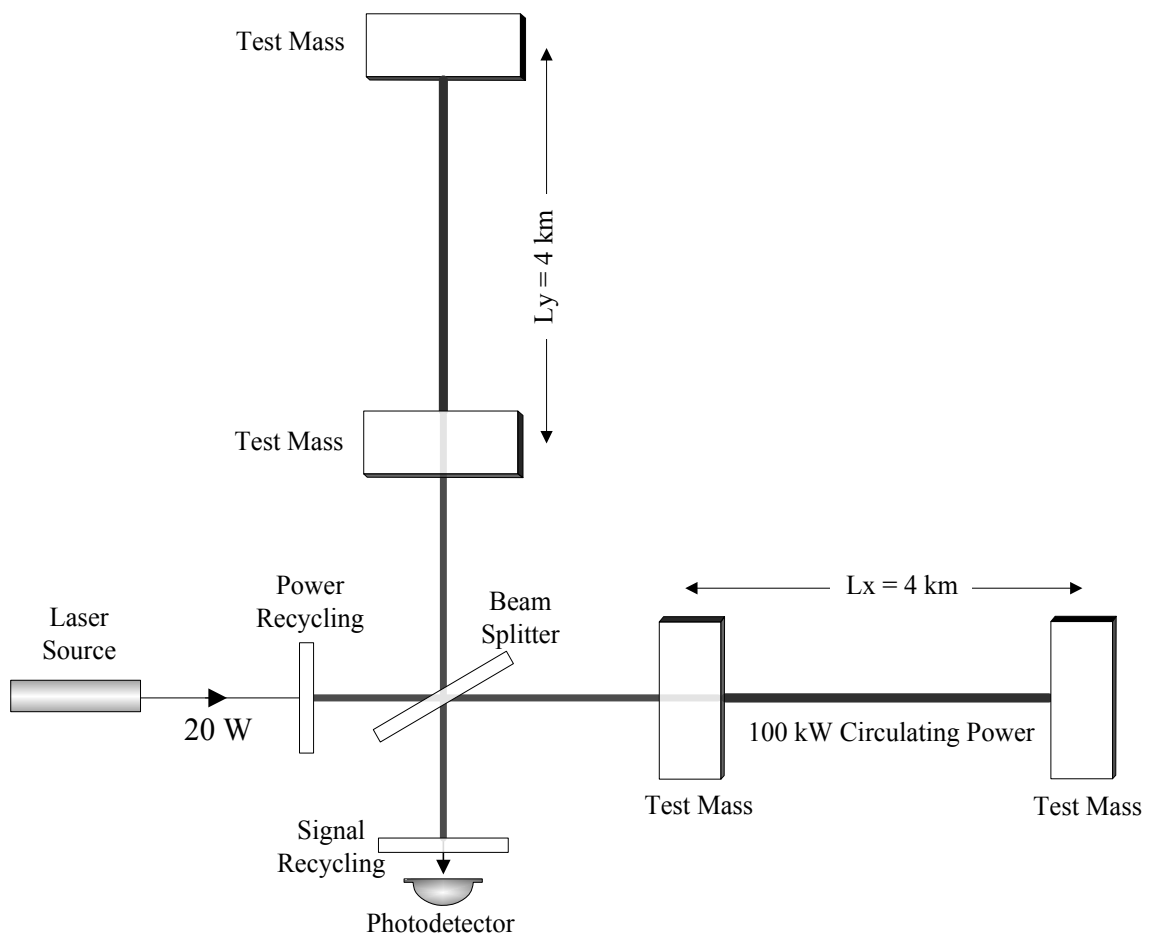

Figure 2. Advanced LIGO detector (see text) (adapted from ref. [49]).

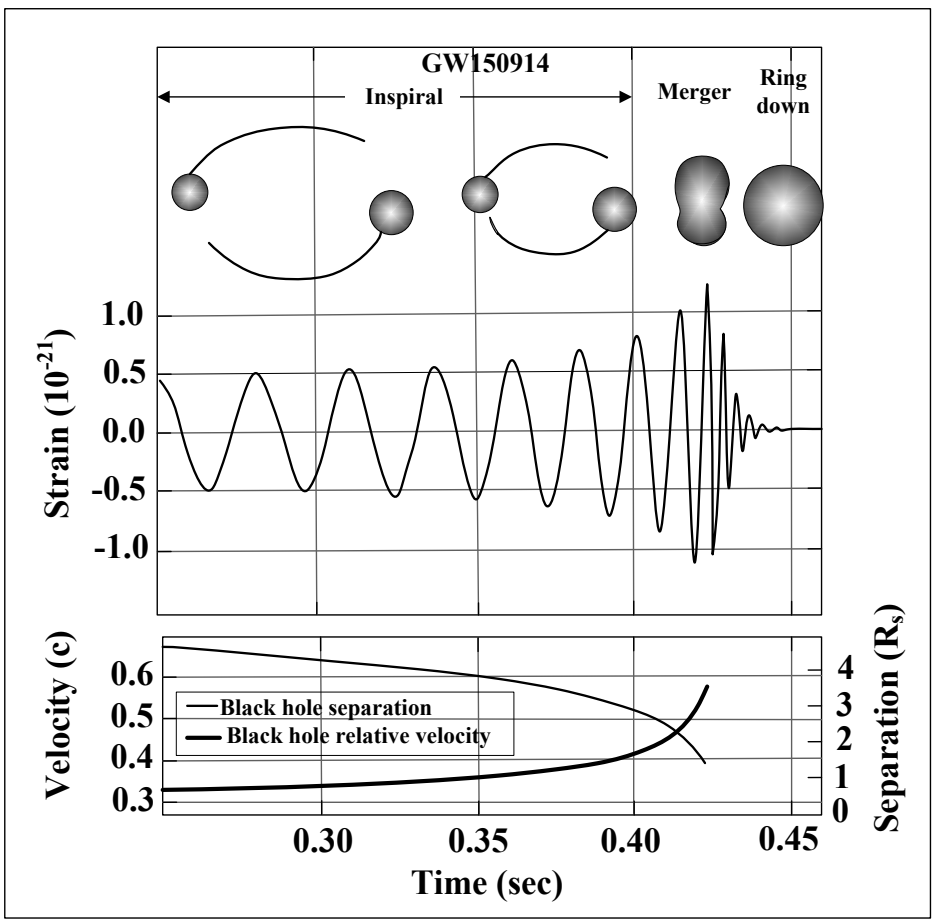

Figure 3. (Top) Estimated gravitation alwave strain amplitude for the GW150914 event. (Bottom) The Keplerian effective black hole separation in units of Schwarzschild radii and the effective relative post-Newtonian parameter $v / c=\left(G M \pi f / c^{3}\right)^{1 / 3}$, where $f$ is the GW frequency calculated numerically, and $M$ is the total mass (adapted from reference [49]). 
[61]. The estimated total energy radiated in gravitational waves in the GW150914 event is $3.0_{-0.5}^{+0.5} M_{\odot} c^{2}$, and the peak gravitational wave luminosity reached a value of $3.6_{-0.4}^{+0.5} \times 10^{56} \mathrm{erg} / \mathrm{s}$, which is equivalent to $200_{-30}^{+30} M_{\odot} c^{2} / \mathrm{s}$. The primary and secondary black holes masse are estimated to be $36_{-4}^{+5} M_{\odot}$ and $29_{-4}^{+4} M_{\odot}$, respectively. The final black hole mass and spin are $62_{-4}^{+4} M_{\odot}$ and $0.67_{-0.07}^{+0.05}$, respectively [49].

Subsequently, gravitational waves emanating from two other binary mergers were discovered. The masses of the black holes for one of the two events is $(14+8) M_{\odot}(G W 151226)[62]$ and for the other $(31+19) M_{\odot}$ [63]. As expected, no electromagnetic (EM) counterpart was discovered despite the massive efforts invested by the astronomical community.

Assuming a modified dispersion relation for the gravitational wave [64], observations constrain the Compton wavelength of the graviton to $\lambda_{g}>10^{13} \mathrm{~km}$, corresponding to a bound graviton mass $m_{g}<1.2 \times 10^{-22} \mathrm{eV} / \mathrm{c}^{2}[53]$.

The GW150914 event demonstrates the existence of stellar-mass black holes with masses exceeding $30 M_{\odot}$, and that binary black holes can form in nature and that they can merge within the Hubble time. These results can be extended to describe what happens when micro black holes collide and merge, as explained in sub-section (8.4).

\subsection{Gravitational Waves from Binary Neutron Star Mergers}

On 17 August 2017 at 12:41:06 UT, the advanced LIGO/Virgo instruments detected the in-spiral and merger of binary neutron stars (GW170817) [56]. The source of the GW was identified as residing in the periphery of the S0 galaxy NGC4993 at a distance of $40 \pm 8 \mathrm{Mpc}(130.4 \pm 26$ million ly $)$ and has a stellar mass of $\log M / M_{\odot}=10.49_{-0.20}^{+0.08} \quad$ [65]. The Swope Supernova Survey 2017a (SSS17a) is the first detection of an electromagnetic counterpart to a gravitational wave source [65]. The observed optical counterpart peaks at an absolute magnitude of $M_{i}=-15.7$, which is 1000 times brighter than a nova [57], or a typical kilonova, as predicted originally by Metzger et al., 2010 [66].

The masses $m_{1}$ and $m_{2}$ of the merging neutron stars are in the range $m_{1} \in(1.36-2.26) M_{\odot}$ and $m_{2} \in(0.86-1.36) M_{\odot}$ [67]. The chirp mass $M_{C}$ drives the frequency evolution of the gravitational radiation in the in-spiral phase (see Equation (7)). The best measured mass parameter is $M_{C}=1.188_{-0.002}^{+0.004} M_{\odot}$. The total mass is $2.82_{-0.09}^{+0.47} M_{\odot}$, and the mass ratio $m_{2} / m_{1}$ is bound to the range $0.4-1.0$ [67]. These masses are consistent with a system of binary neutron stars. White dwarfs are excluded since the gravitational wave signal sweeps through the $200 \mathrm{~Hz}$ frequency in the instruments' sensitivity band, implying an orbit of about $100 \mathrm{~km}$, which is much smaller than the typical radius of a white dwarf [68]. However, for this specific merging event, the gravitational wave data alone cannot exclude more compact objects than neutron stars, such as quark stars or black holes [67]. In the latter case, no EM component is expected to be observed, in violation of the observational evidence. 
A short-lived gamma ray burst (sGRB) was observed independently in the same sky direction by Fermi-GBM [69], and INTEGRAL [59]. A more detailed description of the processes involved in generating and observing sGRB by the merging of NSs is given in sub-section (7.2.3).

Gravitational waves alone can provide only limited information about the nature of the merging progenitors. This is the case for $\mathrm{BH}-\mathrm{BH}$ mergers and for $\mathrm{BH}-\mathrm{NS}$ mergers when the mass of the NS is much less than the mass of the $\mathrm{BH}$ (see Section 3). NS-NS mergers, on the other hand, produce electromagnetic waves (EM), and when combined with GW observations, will provide a wealth of information about the nature and dynamics of the merging NS, the processes involved during the in-spiral, merger, and ringdown phases, as well as the afterglow of the shattered debris following the merger.

Gravitational wave emission probes the bulk motions, binary properties, masses, and hypothetically the composition of the NS. Electromagnetic observations, on the other hand, provide a better understanding of the astrophysics behind the event, particularly the environment of the merging compact objects, the formation of relativistic and non-relativistic outflows, and in certain cases the properties of the merging products [70] [71]. The combined measurements of GW and EM emissions permit new types of measurements, such as the Hubble constant [72] [73] and also provide rich information about the origin of short-lived gamma-ray bursts [75] (see sub-Section 7.2.3).

\subsubsection{UV, Optical, and Near Infrared Transient Afterglow Emissions}

Photometry is a powerful tool for probing a wide range of spectral emissions, and the resulting energy distribution inferred from this photometry can be described by a blackbody model with a well-defined temperature, a radius corresponding to an expansion velocity, and the bolometric luminosity [76]. The afterglow of the merging neutron star binary gives rise to a multi-component spectral energy distribution (SED) across the optical and NIR. It is characterized by a rapid fading of the UV and the blue optical bands, in addition to a significant reddening of the optical/NIR colors [76]. In the optical/NIR bands, the most auspicious counterpart is the kilonova. This is a roughly isotropic thermal transient powered by radioactive decay of rapid neutron capture elements synthesized in the merger ejecta [66] [77] [78] [79] [80]. The luminosity, timescale, and spectral peak of the kilonova depend precisely on the ejecta composition. For ejecta rich with the Fe group or light $r$-process nuclei with atomic mass number $A \leq 140$, the kilonova emission is expected to have a peak at optical wavelengths at a luminosity $L_{p} \approx 10^{41}-10^{42} \mathrm{erg} \cdot \mathrm{s}^{-1}$ on a short timescale of $t_{p} \approx 1$ day, the so-called blue kilonova [66] [78] [81]. On the other hand, for ejecta rich with heavier lanthanide elements $(A \geq 140)$ the emission is predicted to exhibit a peak at NIR wavelengths with $L_{p} \approx 10^{40}-10^{41} \mathrm{erg} \cdot \mathrm{s}^{-1}$ over a longer time scale of $t_{p} \approx 1$ week, which is known as a red kilonova [79] [80] [82]. It was shown that the data for the GRB170817 NS merger event cannot be fitted with a model with heating from ${ }^{56} \mathrm{Ni}$ radioactive decay and Fe-peak opacities, as in normal 
supernovae [76]. However, heating from the $r$-process nuclei requires at least two components and is consistent with lanthanide-poor and lanthanide-rich opacities [76]. In a multi-component model, each component is considered as arising from distinct regions in the ejecta. The high velocity of the blue $\mathrm{KN}$ ejecta suggests that it originates from the shock-heated polar region created when the neutron stars collide (e.g., [83] [84]). On the other hand, the low velocity red $\mathrm{KN}$ component could originate from the vividly ejected tidal tails in the equatorial plane of the binary (e.g., [71] [85] [86]). In this case, the relatively high ejecta mass $\approx 0.01 M_{\odot}$ suggests an asymmetric mass ratio of the emerging binary $(q \leq 0.8,[86])$. The fitted opacity indicates that the hyper-massive neutron star remnant is relatively short-lived $\approx 30 \mathrm{~ms}$, [87] [88].

Models of comprehensive UV, optical, and NIR data for the 170,817 merger event indicate that the total ejecta mass is $\approx 0.05 M_{\odot}$ with a high velocity, $v \approx 0.3 c$, blue component and a slower, $v \approx 0.1-0.2 c$, purple/red component. The presence of both components and the large ejecta mass indicates that binary neutron star mergers are a dominant factor in the cosmic $r$-process nucleosynthesis.

\subsubsection{X-Ray Emission from Neutron Stars Binary Merger}

The Chandra X-ray observatory was successful in detecting the afterglow of GRB170817 at 16 days post trigger. X-ray and radio emission were discovered at the transient's position about 9 and 16 days after the merger. These emissions are likely produced by processes distinct from the ones generating the UV/optical/near infrared emission [87]. The detected luminosity of the X-ray emission from SSS17a has a value of $L(0.3-10 \mathrm{keV})=2.6_{-0.4}^{+0.5} \times 10^{38} \mathrm{erg} \cdot \mathrm{s}^{-1}$, and it follows a power law spectrum with an exponent $\Gamma=2.4 \pm 0.8$ [89]. Moreover, it was found that the X-ray light curve from the binary NS merger associated with the source is consistent with the afterglow from the off-axis short-lived gamma-ray burst, with a jet angle $\geq 23^{\circ}$ from the line of sight [89]. The radio and X-ray data can be jointly explained as the afterglow emission from a sGRB with a jet energy of $10^{49}-10^{50} \mathrm{erg}[90]$.

\subsubsection{Short-Lived Gamma Ray Bursts}

On August 17, 2017, the Fermi Gamma-ray Burst Monitor (GBM) detected a short gamma-ray burst approximately $1.7 \mathrm{~s}$ after the arrival of the gravitational wave that originated from the binary neutron star merger event (170817).

The standard model of short-lived gamma ray bursts (sGRB) predicts that their prompt emission fades on a time scale $<2 \mathrm{~s}$, and that the relativistic jet will be decelerated by the ambient medium. Usually, the jets launched by sGRBs are collimated and highly relativistic; implying that they are only detectable within a narrow range of viewing angles at early times [91]. However, the GRB jet emanating from the GW170817 event was observed off axis and gave rise to a faint afterglow emission at early times that grew in luminosity as the jet beaming became less severe, and its opening angle spread into the line of sight of the ob- 
server [92]. GRB 170817A, which emanated from the 170817 NS merger event, has a duration of $100 \mathrm{~ms}$ as detected by INTEGRAL, and thus it can be assigned to the short-lived GRB class [93]. An important feature of this gamma-ray burst (GRB 170817A) is the low luminosity of its prompt $\gamma$-ray emission. The low luminosity may be due to the off-axis observation of the event. The minimum luminosity of short-lived gamma-ray bursts is not well-constrained observationally, making it difficult to definitely distinguish the luminosity of off-axis GRBs from faint on-axis GRBs.

The off-axis GRB model predicts other multi-wavelength properties, such as late-time radio emission from the afterglow that peaks on timescales of about 10 days after attaining the X-ray peak [92]. Indeed, the radio source associated with SSS17a was reported almost 15 days post-burst [92]. The $\gamma$-ray emission along our line of sight was certainly feeble, or the emission might be deficient in all directions as in the case of low-luminosity long-lived GRBs associated with Ic supernova [94]. This weak burst is thousands of millions of times fainter than the inferred energies of short-lived GRBs and could belong to a separate population of weakly jetted, low luminosity GRBs [95].

The absence of a normal GRB afterglow (e.g., in X-rays; [96]), led certain investigators to suggest the possibility of an off-axis emission mechanism, such as may be produced by a shocked cocoon around the primary jet (e.g., [97] [98]).

The energy spectrum of GRB 170817A is well described by a power law with an exponential cutoff at $\approx 185 \mathrm{KeV}$ [99], and it has a total energy that is about 4 to 6 orders of magnitude less than a typical $S$ wift sGRB [100].

\subsubsection{On the Origin of Heavy Elements}

Colliding neutron stars produce neutron-rich ejecta that can trigger the enhanced $r$-process and gives rise to the so-called kilonova explosion, which powered by the radioactive decay of elements synthesized in the outflow [101]. It has been argued that the high opacity of synthesized heavy elements in the ejecta, such as lanthanides and actinides, will suppress their emission in the optical and it will appear predominantly in the near-infrared on a time scale of several days [80] [101] [102]. The $r$-process is responsible for about half of the elements heavier than iron and has been traditionally attributed to core collapse supernovae [103]. Recent studies, however, disapprove supernovae as a source of at least the heaviest elements of the "platinum peak" near the mass $A=195$ [104]. Nucleosynthesis calculations have confirmed the suitability of compact binary mergers as a source of production of the heaviest elements in the Universe [88] [105] [106] [107] [108]. Unlike colliding black holes, merging neutron stars expel metallic and radioactive debris that can be observed by ground-based telescopes or satellites. As mentioned in sub-section (7.2.1), the modeling of the multi-band light curves, leads to the presence of at least two emission components [104]. The first is characterized by a high opacity and is interpreted as being the tidal part of the dynamical ejecta, carrying a very low electron fraction $\left(\gamma_{e}<0.25\right)$ and resulting in a strong $r$-process that produces lanthanides/actinides [104]. The second component has a low opacity and is characterized by a weaker $r$-process 
via a raised electron fraction $\left(\gamma_{e}>0.25\right)$. This second component may be produced by a different mechanism, such as a neutrino driven wind, and/or the unbinding of the accretion torus material [104]. Finally, these findings provide strong observational evidence that NS binary mergers can resolve the stunning and heated debate on the cosmic origin of heavy and precious elements, such as gold, silver, and platinum.

\section{Gravitational Waves: A Future Perspective}

The LIGO and Virgo instruments have revolutionized our understanding of one of the most challenging phenomena in astrophysics. The scientific community had to wait for about a century to finally witness the detection of gravitational waves predicted originally by the theory of general relativity in 1916. It is worthwhile at this stage to elucidate the lessons we have learned from the first generation of detected gravitational wave events and to investigate the possible impacts of future discoveries of gravitational waves on our vision about the working mechanisms in our universe.

\subsection{Evaluation of Gravitational Waves Detection Events}

The first generation of detected gravitational waves emanating from the merger of compact binaries involved five events, four of which were produced by the merger of binary black holes and only one was produced by the merger of neutron stars. It is to be noted that no black hole-neutron star event has been detected so far. However, it is difficult at this stage to statistically draw any conclusion regarding the frequency of occurrence of each type of binary system. This is basically because black hole binaries are more massive than neutron star binaries and, accordingly, the strain associated with gravitational waves resulting from their merger can be detected to large distances. The most powerful detected event so far involved the merger of a black hole binary with masses of 36 and $29 M_{\odot}$ and a distance of about one billion light years. In comparison, in a sphere of this radius, a limited number of NS-NS binary mergers can be detected. The various types of binary systems can be statistically significant only within a sphere of radius where all these types are potentially detectable. The relative number of the various types of compact binaries can then shed light on the accuracy of the models regarding their evolutionary pathways.

An important outcome of NS-NS merger events is the peculiarity of the short-lived gamma-ray burst (sGRB) typically associated with such events. Their general features are different from most short-lived gamma-ray bursts detected by the $S$ wift satellite. Future detections of GW events emanating from NS-NS mergers may constitute a statistically significant sample that could be used to elucidate the various origins of sGRBs.

\subsection{Primordial Gravitational Waves from the Inflationary Era}

An important prediction of the cosmological inflation theory is the generation of primordial gravitational waves [109]. The amplitude of the GW signal is charac- 
terized by a ratio $(r)$ between the tensor and scalar power spectrum amplitudes at a given pivot scale $k$ [110]. The best bound on $r$ obtained so far yields $r<0.07$ at $95 \%$ confidence level, for $k=0.05 \mathrm{Mpc}^{-1}$ [110]. An important issue is that various inflationary scenarios predict different values of $r$. Therefore, the observation of a gravitational signature of primordial GWs provides a powerful tool to examine the validity of the general inflationary theory and helps also to discriminate between specific inflationary models. Studying the primordial GW background may also probe a wide class of phenomena related to cosmological inflation. For example, the presence of additional field besides the inflaton can generate an extra GW background, not pertaining to vacuum oscillations [111] [112] [113]. A point of interest is the generation of the GW background during the inflationary heating phase [114], which provides a potential test for studying the mechanisms involved in this process. Detection of primordial GWs also has a direct impact on our understanding of fundamental physics. In fact, the energy scale of inflation is intimately related to the tensor-to-scalar ratio $(r)$, whose detection may lead to the exploration of new horizons of physics beyond the standard model of particle physics. It is to be noted that at the early stages of the universe, processes other than inflation may have provided a source for GWs, including the electroweak phase transition [115], the first order phase transition [116] [117], and the topological defects [118] [119]. For all the above reasons, the detection of primordial GWs may shed light on major problems in cosmology in the forthcoming decade. The crucial observational signature of the inflationary GW background is a curl-like pattern in the polarization of the Cosmic Microwave Background (CMB), also referred to as the B-mode. Technological advances in the past decade have steered cosmology towards an exhilarating era where scientists can assess experimentally the existing theoretical models concerning our universe. A number of ground-based or balloon-borne experiments have been designed to probe the B-mode signal. These experiments include Polarbear [120], Piper [121], ACTpol [122], Spider [123], and CLASS [124]. Furthermore, satellites such as COBE, WMAP, and Planck have provided bounds on $r$, as reported above. In addition, several next generation CMB space missions have been planned to detect gravitational waves, and more specifically the B-mode. Among this new generation of missions are COrE [125], LiteBIRD [126], PIXIE [127], and PRISM [128]. Evidence of primordial GWs could also originate from galaxy and CMB curl-like lensing signatures induced by tensor modes [129] [130]. Other signatures of primordial GWs include parameters related to the small modification in the expansion history of the universe arising from GW contributions to the overall energy budget [131]. Finally, a direct detection by future aLIGO [132] or eLISA [133] [134] is a viable possibility, especially if some specific inflationary mechanism generates a blue-tilted primordial tensor spectrum [109].

\subsection{Gravitational Waves from the Relic of Galaxy Formation}

Gravitational waves may have been produced prolifically during the initial phase 
of galaxy formation at the early universe. Several models have been proposed to explain the formation of massive black holes at the center of galaxies at redshifts corresponding to the era when the universe was less than one billion years old. Masses of black holes at the center of faraway galaxies can be estimated using the M-sigma relation [135]. An important question to ask is at what epoch did the seeding of black holes at the centers of galaxies take place, and what are the possible mechanisms involved in their growth. Many hypotheses have been advanced to elucidate the origin of massive or supermassive black holes at the centers of galaxies. Several possible formation channels have been proposed for the formation of massive black hole seeds, including the collapse of population III stars [136], gas dynamic instabilities where supermassive black holes are postulated to form directly out of the dense gas cloud [137] [138], the collapse of supermassive stars [139], and the merger of small and intermediate mass black holes. There is little dispute that once a black hole forms at the center of a galaxy, it can grow either by accretion of surrounding material or by merging with other black holes. In the latter case, gravitational waves will be generated, and if strong enough, they will fall in the domain of sensitivity of the current or future GWs detectors. This merging process is also more likely to happen when galaxies collide to form a cD galaxy [139] [140]. The collision of massive or supermassive black holes at the center of these galaxies will produce energetic GWs that may lie within a sphere of radius, again, corresponding to the sensitivity of the actual or future GWs detectors.

Primordial black holes ( $\mathrm{PBH}$ ) of a wide range of masses have been proposed to form in the extreme densities and inhomogeneities of the early universe [141]. More recently, Choptuik [142] and Kim [143] have demonstrated the formation of PBHs during the inflationary era, when the density of the universe experienced a dramatic decrease causing a cosmological phase transition. In this context, $\mathrm{PBHs}$ could be the seeding agent at the center of the first generation of galaxies in the early universe. Eventually, when the sensitivity of the gravitational wave detectors becomes capable of detecting GW events at redshifts beyond the era of star formation, it is possible that the source of GWs may be shown to be primordial in origin. Studies have shown that if the observed event rate is greater than one event per year at redshifts $z \geq 40$, then the probability distribution of primordial density fluctuations must be significantly non-Gaussian or the events may originate from primordial black holes [144]. Thus, GWs are promising tools that will refine our understanding of the workings of the universe.

\subsection{Quantum Mechanical Black Holes}

In this last section we investigate the possibility of the creation and merger of micro or mini black holes. These are hypothetical entities subject to the rules of quantum mechanics. The concept of micro black holes was initially introduced by Stephen Hawking in 1971 [141]. They could have been created in the high-density environment in the early universe, or possibly through subsequent 
phase transitions, and therefore they are referred to as primordial micro black holes. In principle, primordial black holes may have any mass above the Planck mass, and are characterized by a Schwarzschild radius $R=2 G M / \mathrm{c}^{2}$, where $G$ is the gravitational constant, $c$ the speed of light, and $M$ the mass of the black hole. Some theoretical models hypothesize the existence of extra dimensions of space, where the strength of gravity increases at a faster pace than in three dimensions. Certain configurations of extra dimensions may lower the Planck scale to the $\mathrm{TeV}$ range, which makes the production of micro black holes in the Large Hadron Collider (LHC) a viable possibility [145] [146]. However, quantum effects will cause micro black holes to evaporate almost instantaneously by Hawking radiation, producing a burst of elementary particles [146]. A holeum is a primordial and a hypothetically stable quantized bound state [147]. It consists of a number of mini black holes bound quantum mechanically. In the case of the merger of these mini black holes due to external perturbations, quantum effects will play a major role, and in this case no gravitational waves are likely to be produced, but instead the merger will most probably give rise to a jet of elementary particles.

\section{Conclusion}

We have presented the theoretical framework characterizing gravitational waves emanating from the merger of compact binaries as derived from general relativity, and it was shown that this framework accurately describes the merger events of compact binaries. Detection of gravitational waves from black hole mergers provides evidence of the existence of a wide range of stellar black hole masses. Merging neutron stars give rise to gravitational waves and electromagnetic counterpart emission. The colliding and merging neutron stars give rise to a kilonova, and the observational data can only be explained by assuming multi-component ejecta that produce emissions covering all parts of the spectrum, from gamma rays to radio waves. The observed short gamma-ray bursts produced in these merging events are extremely faint as compared to the ones recorded by the Swift satellite, and this result has cast doubt on the origin of short gamma-ray bursts. A confirmation of the nature of short gamma-ray bursts from merging neutron stars may have to await the discovery of other merging events before a solid model can be set. Analysis of the electromagnetic counterpart of GW170817 reveals that NS-NS mergers are the main source of heavy elements in the universe. The future detection of more GWs will undoubtedly provide a wealth of information concerning the processes that shape our universe.

\section{Conflicts of Interest}

The authors declare no conflicts of interest regarding the publication of this paper. 


\section{References}

[1] Heaviside, O. (1893) Electromagnetic Theory, 1, 455-466.

[2] Poincaré, H. (1905) Note: Sur la dynamique de lélectron. C.R. T, 140, 1504-1508.

[3] Einstein, A. (1916) Prussian Academy Proceedings, 1, 688-696.

[4] Einstein, A. (1918). Prussian Academy Proceedings, 1, 154-167.

[5] LIGO Scientific Collaboration; Virgo Collaboration (2012) Physical Review D, 85, Article ID: 082002.

[6] Hulse, R.A. and Taylor, J.H. (1975) The Astrophysical Journal Letters, 195, L51-L53. https://doi.org/10.1086/181708

[7] Faber, J.A. and Rasio, F.A. (2012) Living Reviews in Relativity, 15, 8. https://doi.org/10.12942/lrr-2012-8

[8] Huang, Y. and Weisberg, J.M. (2016) The Astrophysical Journal, 829, Article ID: 55.

[9] Blanchet, L., et al. (1995) Physical Review Letters, 74, 3515. https://doi.org/10.1103/PhysRevLett.74.3515

[10] Belczynski, K., et al. (2008) The Astrophysical Journal Supplement, 174, 223-260. https://doi.org/10.1086/521026

[11] Bethe, H.A., Brown, G.E. and Lee, C.-H. (2007) Physics Reports, 442, 5-22. https://doi.org/10.1016/j.physrep.2007.02.004

[12] Brown, G.E., Lee, C.-H. and Rho, M. (2008) Physical Review Letters, 101, Article ID: 091101.

[13] Postnov, K. and Yungelson, L. (2006) Living Reviews in Relativity, 9, 6.

[14] Davis, P.J., Kolb, U. and Willems, B. (2010) Monthly Notices of the Royal Astronomical Society, 403, 179-195. https://doi.org/10.1111/j.1365-2966.2009.16138.x

[15] Zorotovic, M., Schreiber, M.R., Gänsicke, B.T. and Nebot Gómez-Morán, A. (2010) Astronomy \& Astrophysics, 520, Article ID: A86.

https://doi.org/10.1051/0004-6361/200913658

[16] Zhang, W.-Q., Woosley, S.E. and Heger, A. (2007) The Astrophysical Journal, 679, 639-654. https://doi.org/10.1086/526404

[17] O’Connor, E. and Ott, C.D. (2011) The Astrophysical Journal, 730, 70. https://arxiv.org/abs/1010.5550

[18] Demorest, P., Pennucci, T., Ransom, S., Roberts, M. and Hessels, J. (2010) Nature, 467, 1081-1083. https://arxiv.org/abs/1010.5788 https://doi.org/10.1038/nature09466

[19] Read, J.S., Lackey, B.D., Owen, B.J. and Friedman, J.L. (2009) Physical Review D, 79, Article ID: 124032. https://arxiv.org/abs/0812.2163

[20] Özel, F. and Psaltis, D. (2009) Physical Review D, 80, Article ID: 103003. https://arxiv.org/abs/0905.1959

[21] Markakis, C., Read, J.S., Shibata, M., Uryū, K., Creighton, J.D.E., Friedman, J.L. and Lackey, B.D. (2009) Journal of Physics. Conference Series, 189, Article ID: 012024. https://arxiv.org/abs/1110.3759

[22] Pandharipande, V.R. and Smith, R.A. (1975) Nuclear Physics A, 237, 507-532. https://doi.org/10.1016/0375-9474(75)90415-7

[23] Wang, C., Lai, D. and Han, J.L. (2006) The Astrophysical Journal, 639, 1007-1017. https://arxiv.org/abs/astro-ph/0509484 https://doi.org/10.1086/499397 
[24] O’Shaughnessy, R., Kim, C., Kalogera, V. and Belczynski, K. (2008) The Astrophysical Journal, 672, 479-488. https://arxiv.org/abs/astro-ph/0610076 https://doi.org/10.1086/523620

[25] Kuranov, A.G., Popov, S.B. and Postnov, K.A. (2009) Monthly Notices of the Royal Astronomical Society, 395, 2087-2094. https://arxiv.org/abs/0901.1055 https://doi.org/10.1111/j.1365-2966.2009.14595.x

[26] Hobbs, G., Lorimer, D.R., Lyne, A.G. and Kramer, M. (2005) Monthly Notices of the Royal Astronomical Society, 360, 974-992.

https://arxiv.org/abs/astro-ph/0504584 https://doi.org/10.1111/j.1365-2966.2005.09087.x

[27] Voss, R. and Tauris, T.M. (2003) Monthly Notices of the Royal Astronomical Society, 342, 1169-1184. https://arxiv.org/abs/astro-ph/0303227

[28] de Freitas Pacheco, J.A., Regimbau, T., Vincent, S. and Spallicci, A. (2006) International Journal of Modern Physics D, 15, 235-250.

https://arxiv.org/abs/astro-ph/0510727 https://doi.org/10.1142/S0218271806007699

[29] Kalogera, V., Belczynski, K., Kim, C., O’Shaughnessy, R. and Willems, B. (2007) Physics Reports, 442, 75-108. https://arxiv.org/abs/astro-ph/0612144 https://doi.org/10.1016/j.physrep.2007.02.008

[30] O’Shaughnessy, R., Kalogera, V. and Belczynski, K. (2010) The Astrophysical Journal, 716, 615-633. https://arxiv.org/abs/0908.3635 https://doi.org/10.1088/0004-637X/716/1/615

[31] Buonanno, A., Chen, Y., Pan, Y., Tagoshi, H. and Vallisneri, M. (2005) Physical Review D, 72, Article ID: 084027. https://arxiv.org/abs/gr-qc/0508064

[32] Babak, S., Balasubramanian, R., Churches, D., Cokelaer, T. and Sathyaprakash, B.S. (2006) Classical and Quantum Gravity, 23, 5477-5504.

https://arxiv.org/abs/gr-qc/0604037

[33] van der Sluys, M., Raymond, V., Mandel, I., Röver, C., Christensen, N., Kalogera, V., Meyer, R. and Vecchio, A. (2008) Classical and Quantum Gravity, 25, Article ID: 184011. https://arxiv.org/abs/0805.1689

[34] Van Den Broeck, C., Brown, D.A., Cokelaer, T., Harry, I., Jones, G., Sathyaprakash, B.S., Tagoshi, H. and Takahashi, H. (2009) Physical Review D, 80, Article ID: 024009. https://arxiv.org/abs/0904.1715

[35] Komatsu, H., Eriguchi, Y. and Hachisu, I. (1989) Monthly Notices of the Royal Astronomical Society, 237, 355-379. https://doi.org/10.1093/mnras/237.2.355

[36] Cook, G.B., Shapiro, S.L. and Teukolsky, S.A. (1992) The Astrophysical Journal, 398, 203-223. https://doi.org/10.1086/171849

[37] Cook, G.B., Shapiro, S.L. and Teukolsky, S.A. (1994) The Astrophysical Journal, 424, 823-845. https://doi.org/10.1086/173934

[38] Baumgarte, T.W., Shapiro, S.L. and Shibata, M. (2000) The Astrophysical Journal Letters, 528, L29-L32. https://arxiv.org/abs/astro-ph/9910565 https://doi.org/10.1086/312425

[39] Shibata, M., Baumgarte, T.W. and Shapiro, S.L. (2000) The Astrophysical Journal, 542, 453-463. https://arxiv.org/abs/astro-ph/0005378 https://doi.org/10.1086/309525

[40] Duez, M.D., Liu, Y.T., Shapiro, S.L., Shibata, M. and Stephens, B.C. (2006) Physical Review Letters, 96, Article ID: 031101. https://arxiv.org/abs/astro-ph/0510653

[41] Stephens, B.C., Duez, M.D., Liu, Y.T., Shapiro, S.L. and Shibata, M. (2007) Classical 
and Quantum Gravity, 24, S207-S220. https://arxiv.org/abs/gr-qc/0610103

[42] Galeazzi, F., Yoshida, S. and Eriguchi, Y. (2012) Astronomy \& Astrophysics, 541, A156. https://arxiv.org/abs/1101.2664

[43] Baumgarte, T.W., Cook, G.B., Scheel, M.A., Shapiro, S.L. and Teukolsky, S.A. (1998) Physical Review D, 57, 7299-7311. https://arxiv.org/abs/gr-qc/9709026 https://doi.org/10.1103/PhysRevD.57.7299

[44] Arvanitaki, A. and Geraci, A.A. (2013) Physical Review Letters, 110, Article ID: 071105.

[45] Shibata, M. and Taniguchi, K. (2011) Living Reviews in Relativity, 14, 6. http://www.livingreviews.org/lrr-2011-6 https://doi.org/10.12942//rr-2011-6

[46] Kyutoku, K., Shibata, M. and Taniguchi, K. (2010) Physical Review D, 82, Article ID: 044049. https://arxiv.org/abs/1008.1460

[47] Faber, J.A., Baumgarte, T.W., Shapiro, S.L., Taniguchi, K. and Rasio, F.A. (2006) Physical Review D, 73, Article ID: 024012. https://arxiv.org/abs/astro-ph/0511366

[48] Thorne, K.S. (1995) Particle and Nuclear Astrophysics and Cosmology in the Next Millennium, 160, 45. https://arxiv.org/abs/gr-qc/9506086 .

[49] Abbott, B.P., et al. (2016) Physical Review Letters, 116, Article ID: 06102.

[50] Drever, R.W.P., et al. (1983) The Advanced LIGO Detectors in the Era of First Discoveries. In: Meystre, P. and Scully, M.O., Eds., Quantum Optics, Experimental Gravity, and Measurement Theory, Vol. 94, Plunum Press, New York, 503-514.

[51] Meers, B.J. (1988) Physical Review D, 38, Article ID: 2317. https://doi.org/10.1103/PhysRevD.38.2317

[52] Kwee, P., et al. (2012) Optics Express, 20, Article ID: 10617.

[53] Fricke, T.T., et al. (2012) Classical Quantum Gravity, 29, Article ID: 065005.

[54] Taracchini, A., et al. (2014) Physical Review D, 89, Article ID: 061505.

[55] Pürrer, M. (2014) Classical Quantum Gravity, 31, Article ID: 195010.

[56] Hannam, M., et al. (2014) Physical Review Letters, 113, Article ID: 151101.

[57] Khan, S., et al. (2016) Physical Review D, 93, Article ID: 044007.

[58] Veitch, J., et al. (2015) Physical Review D, 91, Article ID: 042003.

[59] Healy, J., et al. (2014) Physical Review D, 90, Article ID: 104004.

[60] Husa, S., et al. (2016) Physical Review D, 93, Article ID: 044006.

[61] Abbott, B.P., et al. (2016) Physical Review D, 93, Article ID: 122004.

[62] Abbott, B.P., et al. (2016) Physical Review Letters, 116, Article ID: 241103.

[63] Abbott, B.P., et al. (2017) Physical Review Letters, 118, Article ID: 221101.

[64] Will, C.M. (1998) Physical Review D, 57, Article ID: 2061.

[65] Pan, Y.C., et al. (2017) The Astrophysical Journal Letters, 848, L30. https://doi.org/10.3847/2041-8213/aa9116

[66] Metzger, B.D., et al. (2010) Monthly Notices of the Royal Astronomical Society, 406, Article ID: 2650.

[67] Abbott, B.P., et al. (2017) The Astrophysical Journal Letters, 848, L12. https://doi.org/10.3847/2041-8213/aa91c9

[68] Shapiro, S.L. and Teukolsky, S.A. (1983) The Physics of Compact Objects. John Wiley, New York.

[69] Goldstein, A., et al. (2017) The Astrophysical Journal Letters, 848, L14. 
https://doi.org/10.3847/2041-8213/aa8f41

[70] Metzger, B.D. and Berger, E. (2012) The Astrophysical Journal, 746, 48. https://doi.org/10.1088/0004-637X/746/1/48

[71] Rosswog, S., Piran, T. and Nakar, E. (2013) Monthly Notices of the Royal Astronomical Society, 430, 2585-2604. https://doi.org/10.1093/mnras/sts708

[72] Schutz, B.F. (1986) Nature, 323, 310-311. https://doi.org/10.1038/323310a0

[73] Holz, D.E. and Hughes, S.A. (2005) The Astrophysical Journal, 629, 15. https://doi.org/10.1086/431341

[74] Nissanke, S., et al. (2010) The Astrophysical Journal, 725, 496. https://doi.org/10.1088/0004-637X/725/1/496

[75] Berger, E. (2014) Annual Review of Astronomy and Astrophysics, 52, 43-105. https://doi.org/10.1146/annurev-astro-081913-035926

[76] Cowperthwaite, P.S., et al. (2017) The Astrophysical Journal Letters, 848, L17. https://doi.org/10.3847/2041-8213/aa8fc7

[77] Li, L.X. and Paczynski, B. (1998) The Astrophysical Journal Letters, 507, L59. https://doi.org/10.1086/311680

[78] Roberts, L.F. (2011) The Astrophysical Journal Letters, 736, L21. https://doi.org/10.1088/2041-8205/736/1/L21

[79] Barnes, J. and Kasen, D. (2013) The Astrophysical Journal, 775, 18. https://doi.org/10.1088/0004-637X/775/1/18

[80] Tanaka, M. and Hotokezaka, K. (2013) The Astrophysical Journal, 775, 113. https://doi.org/10.1088/0004-637X/775/2/113

[81] Metzger, B.D. and Fernandez, R. (2014) Monthly Notices of the Royal Astronomical Society, 441, 3444-3453. https://doi.org/10.1093/mnras/stu802

[82] Kasen, D., Badnell, N. and Barnes, J. (2013) The Astrophysical Journal, 774, 25. https://doi.org/10.1088/0004-637X/774/1/25

[83] Bauswein, A., et al. (2013) The Astrophysical Journal, 773, 78. https://doi.org/10.1088/0004-637X/773/1/78

[84] Sekiguchi, Y., et al. (2016) Physical Review D, 93, Article ID: 124046.

[85] Rosswog, S., et al. (1999) Astronomy and Astrophysics, 341, 499.

[86] Hotokezaka, K., et al. (2013) Physical Review D, 87, Article ID: 024001.

[87] Fernandez, R. and Metzger, B. D. (2013) Monthly Notices of the Royal Astronomical Society, 435, 502-517. https://doi.org/10.1093/mnras/stt1312

[88] Just, O., et al. (2015) Monthly Notices of the Royal Astronomical Society, 48, 541-567. https://doi.org/10.1093/mnras/stv009

[89] Hagard, D., et al. (2017) The Astrophysical Journal Letters, 848, L25. https://doi.org/10.3847/2041-8213/aa8ede

[90] Alexander, K.D., et al. (2017) The Astrophysical Journal Letters, 848, L21. https://doi.org/10.3847/2041-8213/aa905d

[91] Fong, W., et al. (2015) The Astrophysical Journal, 815, 102. https://doi.org/10.1088/0004-637X/815/2/102

[92] Hallinan, G., et al. (2017) Science, 358, 1579-1583.

[93] Savchenko, V., et al. (2017) The Astrophysical Journal Letters, 848, L5. https://doi.org/10.3847/2041-8213/aa8f94

[94] Kaneko, Y., et al. (2007) The Astrophysical Journal, 654, 385. 
https://doi.org/10.1086/508324

[95] Murguia-Berthier, A., et al. (2017) The Astrophysical Journal Letters, 848, L34. https://doi.org/10.3847/2041-8213/aa91b3

[96] Evans, P.A., et al. (2017) Science, 358, 1565-1570. https://doi.org/10.1126/science.aap9580

[97] Gottieb, O., Nakar, E. and Piran, T. (2017) Monthly Notices of the Royal Astronomical Society, 473, 576-584.

[98] Lazzati, D., et al. (2017) Monthly Notices of the Royal Astronomical Society, 471, 1652-1661. https://doi.org/10.1093/mnras/stx1683

[99] LIGO Scientific Collaboration and Virgo Collaboration (2017) The Astrophysical Journal Letters, 848, L13. https://doi.org/10.3847/2041-8213/aa920c

[100] Gehrels, N., et al. (2009) Annual Review of Astronomy and Astrophysics, 47, 567-617. https://doi.org/10.1146/annurev.astro.46.060407.145147

[101] Pian, E., et al. (2017) Spectroscopic Identification of r-Process Nucleosynthesis in a Double Neutron Star Merger. arXiv:1710.05858

[102] Arcavi, I., et al. (2017) Nature, 551, 64-66.

[103] Burbidge, E.M., et al. (1957) Review of Modern Physics, 29, 547. https://doi.org/10.1103/RevModPhys.29.547

[104] Tanvir, N.R., et al. (2017) The Astrophysical Journal Letters, 848, L27. https://doi.org/10.3847/2041-8213/aa90b6

[105] Rosswog, S., et al. (1998) Astronomy and Astrophysics, 341, 499-526.

[106] Freiburghaus, C., et al. (1999) The Astrophysical Journal Letters, 525, L121. https://doi.org/10.1086/312343

[107] Goriely, S., et al. (2011) The Astrophysical Journal, 738, L32. https://doi.org/10.1088/2041-8205/738/2/L32

[108] Mendosa-Temis, J.D.J., et al. (2015) Physical Review C, 92, Article ID: 055805.

[109] Guzzetti, M.C., et al. (2016) La Rivista del Nuovo Cimento, 39, 399-495.

[110] Ade, P.A.R., et al. (2016) Physical Review Letters, 116, Article ID: 031302.

[111] Barnaby, N., et al. (2012) Physical Review D, 86, Article ID: 103508.

[112] Binetruy, P., et al. (2012) JCAP, 1206, 27.

[113] Biagetti, M., et al. (2013) Physical Review D, 88, Article ID: 103518.

[114] Khlebnikov, S.Y. and Tkachev, I.I. (1996) Physical Review Letters, 77, 219. https://doi.org/10.1103/PhysRevLett.77.219

[115] Huber, S.J., et al. (2016) JCAP, 1604, 36.

[116] Caprini, C., et al. (2016) JCAP, 1604, 1.

[117] Dev, P.S.B. and Mazumdar, A. (2016) Physical Review D, 93, Article ID: 104001.

[118] Sanidas, S.A., et al. (2012) Physical Review D, 85, Article ID: 122003.

[119] Figueroe, D.G., et al. (2013) Physical Review Letters, 110, Article ID: 101302.

[120] Ade, P.A.R., et al. (2014) The Astrophysical Journal, 794, Article ID: 171.

[121] Gandilo, N.N., et al. (2016) PIPER, 1607, Article ID: 06172.

[122] Calabrese, E., et al. (2014) JCAP, 1408, 10.

[123] Crill, B.P., et al. (2008) Proceedings of SPIE-The International Society for Optical Engineering, 7010, Article ID: 08071548.

[124] Eimer, J.R., et al. (2012) Proceedings of SPIE-The International Society for Optical 
Engineering, 8452, Article ID: 12110041.

[125] Bouchet, F.R., et al. (2011) https://arxiv.org/abs/1102.2181

[126] Matsumura, T., et al. (2013) Journal of Low Temperature Physics, 176, 733. https://doi.org/10.1007/s10909-013-0996-1

[127] Kogut, A., et al. (2011) JCAP, 1107, 25.

[128] Andrè, P., et al. (2014) JCAP, 1402, 6.

[129] Dodelson, S., et al. (2003) Physical Review Letters, 91, Article ID: 021301. https://arxiv.org/abs/astro-ph/0301177

[130] Jeong, D. and Schmidt, F. (2012) Physical Review D, 86, Article ID: 083512.

[131] Smith, T.L., et al. (2006) Physical Review Letters, 97, Article ID: 021301. https://arxiv.org/pdf/astro-ph/0603144v1.pdf

[132] Aasi, J., et al. (2015) Classical and Quantum Gravity, 32, Article ID: 074001.

[133] eLISA Science Collaboration. https://www.elisascience.org/

[134] Klein, A., et al. (2016) Physical Review D, 93, Article ID: 024003.

[135] Ferrarese, F. and Merrit, D. (2000) The Astronomical Journal, 539, L9-L12. https://doi.org/10.1086/312838

[136] Bromm, V., et al. (1999) The Astrophysical Journal Letters, 527, L5-L8. https://doi.org/10.1086/312385

[137] Haehnelt, M.G. and Rees, M.J. (1993) Monthly Notices of the Royal Astronomical Society, 263, 168-178. https://doi.org/10.1093/mnras/263.1.168

[138] Lodato, G. and Natarajan, P. (2006) Monthly Notices of the Royal Astronomical Society, 371, 1813-1823. https://doi.org/10.1111/j.1365-2966.2006.10801.x

[139] Volonteri, M. (2010) The Astronomy and Astrophysics Review, 18, 279-315. https://doi.org/10.1007/s00159-010-0029-x

[140] Leahy, J.P. (1997) An Atlas of DRAGNs, "Glossary".

[141] Hawking, S.W. (1971) Monthly Notices of the Royal Astronomical Society, 152, 75-78. https://doi.org/10.1093/mnras/152.1.75

[142] Choptuik, M.W. (1993) Physical Review Letters, 70, 9-12. https://doi.org/10.1103/PhysRevLett.70.9

[143] Kim, H.I. (2000) Physical Review D, 62, Article ID: 063504.

[144] Koushiappas, S.M. and Loeb, A. (2017) Physical Review Letters, 119, Article ID: 221104.

[145] Giddings, S.B. and Thomas, S.D. (2002) Physical Review D, 65, Article ID: 056010.

[146] Dimopoulos, S. and Landsberg, G.L. (2001) Physical Review Letters, 87, Article ID: 161602.

[147] Chavda, L.K. and Chavda, A.L. (2002) Classical and Quantum Gravity, 19, 11. https://doi.org/10.1088/0264-9381/19/11/311 